University of Texas at El Paso

ScholarWorks@UTEP

8-2019

\title{
Status Quo Bias Actually Helps Decision Makers to Take Nonlinearity into Account: An Explanation
}

\author{
Griselda Acosta \\ The University of Texas at El Paso, gvacosta@miners.utep.edu \\ Eric Smith \\ The University of Texas at El Paso, esmith2@utep.edu \\ Vladik Kreinovich \\ The University of Texas at El Paso, vladik@utep.edu
}

Follow this and additional works at: https://scholarworks.utep.edu/cs_techrep

Part of the Applied Mathematics Commons, and the Computer Sciences Commons Comments:

Technical Report: UTEP-CS-19-91

\section{Recommended Citation}

Acosta, Griselda; Smith, Eric; and Kreinovich, Vladik, "Status Quo Bias Actually Helps Decision Makers to Take Nonlinearity into Account: An Explanation" (2019). Departmental Technical Reports (CS). 1357. https://scholarworks.utep.edu/cs_techrep/1357

This Article is brought to you for free and open access by the Computer Science at ScholarWorks@UTEP. It has been accepted for inclusion in Departmental Technical Reports (CS) by an authorized administrator of ScholarWorks@UTEP.For more information, please contact Iweber@utep.edu. 


\title{
Status Quo Bias Actually Helps Decision Makers to Take Nonlinearity into Account: An Explanation
}

\author{
Griselda Acosta ${ }^{1}$, Eric Smith ${ }^{2}$, and Vladik Kreinovich ${ }^{3}$ \\ ${ }^{1}$ Department of Electrical and Computer Engineering \\ ${ }^{2}$ Department of Industrial, Manufacturing, and \\ Systems Engineering \\ ${ }^{3}$ Department of Computer Science \\ University of Texas at El Paso \\ 500 W. University \\ El Paso, TX 79968, USA \\ gvacosta@miners.utep.edu, esmith2@utep.edu,vladik@utep.edu
}

\begin{abstract}
One of the main motivations for designing computer models of complex systems is to come up with recommendations on how to best control these systems. Many complex real-life systems are so complicated that it is not computationally possible to use realistic nonlinear models to find the corresponding optimal control. Instead, researchers make recommendations based on simplified - e.g., linearized - models. The recommendations based on these simplified models are often not realistic but, interestingly, they can be made more realistic if we "tone them down" - i.e., consider predictions and recommendations which are close to the current status quo state. In this paper, we analyze this situation from the viewpoint of general system analysis. This analysis explain the above empirical phenomenon - namely, we show that this "status quo bias" indeed helps decision makers to take nonlinearity into account.
\end{abstract}

\section{Formulation of the Problem}

Real-life problems. In his presentation [1] at the 2019 World Congress of the International Fuzzy Systems Association (IFSA), Professor Kacprzyk recalled his experience of optimizing large systems - like an economic region - at the International Institute for Applied Systems Analysis (IIASA) in Laxenburg, Austria.

Linearization is needed. Of course, many dependencies in complex real-life systems are non-linear. However, even with modern computers, optimizing a 
complex system under nonlinear constraints would require an unrealistic amount of computation time. As a result, in the optimization, most processes in a reallife system were approximated by linear models.

Comment. To be more precise, when analyzing the effect of one specific strategy on a system, we can afford to take non-linearity into account. However, when we need to solve an optimization problem of selecting the optimal strategy and/or optimal parameters of such a strategy, we have to use linearization.

Recommendations based on the linearized model were often not realistic. Not surprisingly, since the optimization process involved simplification of the actual system, recommendations based on the resulting simplified model were often not realistic and could not be directly implemented.

This was not just a subjective feeling: when the researchers tested, on the nonlinear model, the effect of a strategy selected based on linearization, the results were often not so good.

Status quo bias helped. One of the reasons that people listed for being reluctant to accept the center's recommendations was that these recommendations differed too much from what they expected. This phenomenon of unwillingness to follow recommendations if they are too far away from the status quo is known as the status quo bias; see, e.g., [2, 3].

Interestingly, when the center's researchers "toned down" their recommendations by making them closer to the status quo, the resulting recommendations led to much better results (e.g., as tested on the nonlinear models).

In other words, the toning down - corresponding to what we understand as the status quo bias - actually improved the decisions in comparison to simply using recommendations based on the simplified linear models. Thus, the status quo bias somehow takes into account non-linearity - and is, thus, not a deviation from an optimal decision making (as the word bias makes you think) but rather a reasonable way to come up with a better decision.

But why? The phenomenon described above seems mysterious. Why would getting closer to the status quo lead to a better solution?

In this paper, we analyze this phenomenon from the general system approach. Our analysis allows us to explain why the status quo bias indeed helps to take some nonlinearity into account.

\section{Analysis of the Problem and the Resulting Explanation}

A general description of a system: a brief reminder. In general, the state of a system at each moment of time $t$ can be described by listing the values $x_{1}(t), \ldots, x_{n}(t)$ of all the quantities $x_{1}, \ldots, x_{n}$ that characterize this system. Similarly, the change in the system can be described by differential equations 
that explain how the value of each of these quantities change with time:

$$
\frac{d x_{i}(t)}{d t}=f_{i}\left(x_{1}(t), \ldots, x_{n}(t)\right), \quad i=1, \ldots, n .
$$

Here the expressions $f_{i}\left(x_{1}, \ldots, x_{n}\right)$ describe how the rate of change in each of the quantities depends on the state $x=\left(x_{1}, \ldots, x_{n}\right)$; in general, the expressions $f_{i}\left(x_{1}, \ldots, x_{n}\right)$ are non-linear.

In particular, in the simplest case when we use the value of only quantity $x_{1}$ to describe the state, we get the equation

$$
\frac{d x_{1}(t)}{d t}=f_{1}\left(x_{1}(t)\right)
$$

What happens when we linearize. When we linearize the description of the system, we thus replace the nonlinear functions $f_{i}\left(x_{1}, \ldots, x_{n}\right)$ by their linear approximations

$$
f_{i}\left(x_{1}, \ldots, x_{n}\right) \approx a_{i}+\sum_{j=1}^{n} a_{i j} \cdot x_{j} .
$$

In particular, in the case when we use only quantity $x_{1}$, we get the following approximate equation

$$
\frac{d x_{1}}{d t}=a_{1}+a_{11} \cdot x_{1} .
$$

In this case, for the auxiliary variable $y_{1} \stackrel{\text { def }}{=} x_{1}+\frac{a_{1}}{a_{1}}$, we get

$$
\frac{d y_{1}}{d t}=a_{11} \cdot y_{1}
$$

The solution to this simple differential equation is well-known: it is

$$
y_{1}(t)=y_{1}(0) \cdot \exp \left(a_{11} \cdot t\right)
$$

and thus,

$$
x_{1}(t)=y_{1}(y)-\frac{a_{1}}{a_{11}}=y_{1}(0) \cdot \exp \left(a_{11} \cdot t\right)-\frac{a_{1}}{a_{11}} .
$$

In situations when the value of $x_{1}$ (and thus, of $y_{1}$ ) decreases, we have $a_{11}<0$. In such situations, the value $y_{1}$ decreases to 0 . Such things happen. However, in situations when we want to describe the growth, i.e., when $a_{11}>0$, we get an exponential growth.

Exponential growth may be a good approximation for some period of time, but eventually it starts growing too fast to be realistic. For example, in good times, economies grow - but we do not expect, e.g., production of meat to grow thousands times. Similarly, pollution grows, or, on a somewhat less negative side, populations grow, but we do not expect thousands-times increases predicted by the exponential models. 
This phenomenon of models-growing-too-fast is not limited to the case when the system if described by only one variable. In general, a solution to a system of linear differential equations with constant coefficients is a linear combination of oscillatory terms and exponential terms - so, if we describe growth, the models will make it unrealistically exponential.

How to make conclusions more realistic. Linear models are not realistic - the deviations from the current values and what these models predict become too large to be realistic.

Thus, a natural way to make the models more realistic is to take this phenomenon into account - i.e., instead of the results of the linearized models, consider states which are closer to the original state

$$
x(0)=\left(x_{1}(0), \ldots, x_{n}(0)\right) .
$$

Conclusion. This idea of considering the states which are closer to the original state than the model suggests is exactly what the status quo bias is about. Thus, indeed, the status quo bias helps take make models more realistic.

The unrealistic character of the linearized model's recommendation is caused by the fact that this model is only approximate - it ignores nonlinear terms. So, by making recommendations more realistic, the status quo bias, in effect, helps us to take nonlinearity into account.

\section{Acknowledgments}

This work was supported in part by the National Science Foundation grants 1623190 (A Model of Change for Preparing a New Generation for Professional Practice in Computer Science) and HRD-1242122 (Cyber-ShARE Center of Excellence).

\section{References}

[1] J. Kacprzyk, "Cognitive biases in choice and decision making: a potential role of fuzzy logic", Proceedings of the Joint World Congress of International Fuzzy Systems Association and Annual Conference of the North American Fuzzy Information Processing Society IFSA/NAFIPS'2019, Lafayette, Louisiana, June 18-21, 2019.

[2] D. Kahneman, J. L. Knetsch, and R. H. Thaler, "Anomalies: the endowment effect, loss aversion, and status quo bias", Journal of Economic Perspectives, 1991, Vol. 5, No. 1, pp. 193-206.

[3] W. Samuelson and R. Zeckhauser, "Status quo bias in decision making", Journal of Risk and Uncertainty, 1988, Vol. 1, pp. 7-59. 